\title{
Relativistic corrections to fractal analyses of the galaxy distribution
}

\author{
M.-N. Célérier ${ }^{1}$ and R. Thieberger ${ }^{2}$ \\ 1 Département d'Astrophysique Relativiste et de Cosmologie, Observatoire de Paris-Meudon, \\ 5 place Jules Janssen, 92195 Meudon Cedex, France \\ 2 Physics Department, Ben Gurion University, Beer Sheva 84105, Israel \\ e-mail: thieb@bgumail.bgu.ac.il
}

Received 3 July 2000 / Accepted 14 November 2000

\begin{abstract}
The effect of curvature on the results of fractal analyses of the galaxy distribution is investigated. We show that, if the universe satisfies the criteria of a wide class of parabolic homogeneous models, the observers measuring the fractal index with the integrated conditional density procedure may use the Hubble formula, without having to allow for curvature, out to distances of $600 \mathrm{Mpc}$, and possibly far beyond. This contradicts a previous claim by Ribeiro (1995) that, in the Einstein-de Sitter case, relativistic corrections should be taken into account at much smaller scales. We state for the class of cosmological models under study, and give grounds for conjecture for others, that the averaging procedure has a smoothing effect and that, therefore, the redshift-distance relation provides an upper limit to the relativistic corrections involved in such analyses.
\end{abstract}

Key words. galaxies: general - relativity

\section{Introduction}

Standard cosmology is based on the assumption that the universe is spatially homogeneous, at least on scales sufficiently large to justify its approximation by a FriedmannLemaitre-Robertson-Walker (FLRW) model. The high isotropy measured in the cosmic microwave background radiation (CMBR) is usually taken as strong evidence in support of this hypothesis. Coupled to a global (local) Copernican principle, this observation has led to the formulation of the Ehlers-Geren-Sachs (Ehlers et al. 1968) (almost Ehlers-Geren-Sachs (Stoeger et al. 1995)) theorems. Although the assumptions underlying these theorems have been discussed by some authors (Clarkson \& Barrett 1999; Célérier 2000a), they are generally considered as robust support for the Cosmological Principle.

The structures seen in galaxy catalogues - groups, clusters and superclusters, distributed along voids, filaments and walls - are not viewed as contradicting this Principle, as the scales on which the universe is assumed to be homogeneous are much larger than those subtended by these structures. Furthermore, the most probable existence of dark matter implies that the luminous matter seen in the galaxies does not represent the entire source of gravitational energy. Thus, the high isotropy of the CMBR

Send offprint requests to: M.-N. Célérier, e-mail: Marie-Noelle.Celerier@obspm.fr could, in principle, be reconciled with an inhomogeneous luminous matter distribution, provided the missing mass for an homogeneous pattern would be provided by dark matter. This important issue, which has been formulated as the problem of finding whether "light traces mass" is still the subject of a number of interesting works (see e.g., Bahcall et al. 2000), but appears far from being settled. However, a majority of cosmologists seems to feel more confortable with the idea that the structure distribution should present a transition towards homogeneity at a more or less large scale.

However, the consensus on a homogeneous feature of structures, even on very large scales, has never been complete. The debate extends back to Newton (pro-homogeneous) versus Kant and Lambert (prohierarchical). The pro-hierarchical approach was reconsidered in a more modern way by Charlier $(1908,1922)$, and later developed by de Vaucouleurs $(1953,1970)$, who exhibited a universal density radius power law. Recent ideas on fractals (Mandelbrot 1982) led to the pioneering work by Pietronero (1987). Since then, improvements in observational techniques have increased the number and depth of available three dimensional galaxy catalogues. A program of systematic analyses of these catalogues has led to the claim that one can identify a fractal distribution up to $150 h^{-1}$ Mpc (Sylos Labini et al. 1998). Although these results are disputed by many authors (see e.g., Kerscher 1999), it is now believed by many others that one has 
to increase substentially the $5 h^{-1} \mathrm{Mpc}$ lower boundary generally accepted in the early eighties for the scale of the homogeneity transition (Davis \& Peebles 1983). The ongoing sharp controversy about this issue awaits a new generation of galaxy catalogues in order to be settled (Murante et al. 1998; Martinez 1999).

One of the assumptions retained by almost every author in the field is that Euclidean geometry may be used as a reasonable approximation, up to the distances reached by the current galaxy catalogues (Peebles 1980; Sylos Labini et al. 1998). This statement has, however, been disputed by Ribeiro (1995), who argued that the usual practice of disregarding relativistic effects in the study of galaxy distribution could cease to be valid beyond much smaller scales than commonly believed. Using the Einstein-de Sitter model as a theoretical framework, he claimed that this approximation could be misleading for the scales probed by the most recent catalogues.

The role of curvature in a universe exhibiting fractal structures has also been explored by Humphrey et al. (1998), using, as a model, an inhomogeneous isotropic bubble matching a FLRW background. Their conclusion was that Einstein's equations and the matching conditions imply either a nonlinear Hubble law or a very low largescale density, provided no selection, evolution or cosmological constant effect should come into play.

Since then, there has been a growth in the literature on this issue. The scale beyond which the relativistic corrections become non-negligible for the power spectrum analyses of redshift surveys has, for instance, been recently explored by de Laix \& Starkman (1998) and Mansouri \& Rahvar (1999). These authors also claim that a light-cone effect is noticeable at lower redshifts than usually believed.

The purpose of the work we present here is to delve deeper into the tests of the sensitivity to relativistic corrections of the methods used in calculating the distribution of galaxies. We show that Ribeiro's claim, applied to the Einstein-de Sitter case, is ill-founded. Extending our study to other homogeneous cosmological models, we show that the Euclidean approximation can be considered as reasonable up to scales larger than currently reached, for a wide class of standard universes, and that, for this peculiar class, the redshift-distance relation applying to a given cosmological model provides an upper limit to the relativistic corrections involved.

In fact, we know that the Friedmann relation between redshift and comoving distance has little chance of being valid in our clumpy universe (see e.g., KurkiSuonio \& Liang 1992; Mustapha et al. 1998; Célérier 2000b). However, in this work, we choose, for simplicity, to study the effect of the averaging procedure mainly used for the completion of fractal analyses on the relativistic corrections, for peculiar (parabolic) FLRW models. We show that, in these models, this procedure has actually a smoothing effect and we provide grounds to conjecture that this effect could apply to any other kind of (more physical) cosmological models.
This article is organised as follows. In Sect. 2, we give a short description of the statistical methods used to quantify the galaxy distribution. Section 3 is devoted to an analytical study of the Einstein-de Sitter case and to a discussion of Ribeiro's results. In Sect. 4, we describe the virtual catalog method employed to complete our numerical simulations, which are presented, with their results, in Sect. 5. A discussion and the conclusion appear in Sect. 6. The analytical expressions used for the calculations are given in the Appendix.

\section{Quantifying the galaxy distribution}

In this section we give a short description of the methods used for quantifying the galaxy distribution. The purpose of this digression is to make the arguments concerning our main theme clearer.

The standard procedure, pioneered by Peebles (1980), was to follow the methods used for gases and liquids. The one-body density, $n$, is assumed to be constant. The twobody density, denoted by $n^{(2)}\left(\boldsymbol{r}^{\prime}, \boldsymbol{r}^{\prime \prime}\right)$, is given by

$n^{(2)}\left(\boldsymbol{r}^{\prime}, \boldsymbol{r}^{\prime \prime}\right)=n^{2} g\left(\left\|\boldsymbol{r}^{\prime \prime}-\boldsymbol{r}^{\prime}\right\|\right)$.

Here $g(r)$ is the pair distribution function. The main interest lies in the deviation of $n^{(2)}$ from independent particle behaviour and so a two point correlation function is defined by $\xi(r)=g(r)-1$. According to the results obtained by Peebles,

$\xi(r)=\left(\frac{r}{r_{0}}\right)^{-\gamma}, \quad r<r_{0}$.

The value for $r_{0}$ was given as $5 h^{-1} \mathrm{Mpc}$.

It was pointed out by Pietronero (1987) that the $r_{0}$ value is a spurious result due to the assumption that $n$ is a constant. From Eq. (1), we see that the density at $\boldsymbol{r}^{\prime \prime}$ under the condition that there is a galaxy at $\boldsymbol{r}^{\prime}$ is given by the conditional density $n^{(1)}\left(\boldsymbol{r}^{\prime \prime}\right) g\left(\left\|\boldsymbol{r}^{\prime \prime}-\boldsymbol{r}^{\prime}\right\|\right)$. If all galaxies are equivalent, we can choose $\boldsymbol{r}^{\prime}=0$ and then,

$n^{(1)}\left(\boldsymbol{r}^{\prime \prime}\right) g\left(\boldsymbol{r}^{\prime \prime}\right)=\frac{n^{(2)}\left(0, \boldsymbol{r}^{\prime \prime}\right)}{n^{(1)}(0)}$.

Assuming isotropy (i.e. all directions to be equivalent), the right hand side of Eq. (3) depends solely on $\left\|\boldsymbol{r}^{\prime \prime}\right\|$, and we can write:

$n^{(1)}(\boldsymbol{r}) g(\boldsymbol{r})=\Gamma(r), \quad r=\|\boldsymbol{r}\|$.

Here $\Gamma$ is defined by the right hand side of Eq. (3). As it has been shown by Pietronero (1987), $\Gamma(r)$ is the physically significant expression, rather than $\xi(r)$. If we can assume $n^{(1)}=$ const., then we can write a relation between $\xi(r)$ and $\Gamma(r)$ :

$\xi(r)=\frac{\Gamma(r)}{n^{(1)}(0)}-1$

Just as in Eq. (2), we can write for $\Gamma$,

$\Gamma(r)=$ const. $r^{-\gamma_{\Gamma}}$. 
It was pointed out by Thieberger et al. (1990) that the analysis of data does not necessarily give the same value for $\gamma$ and $\gamma_{\Gamma}$. Taking a set of points from a known fractal and performing these calculations showed that the theoretical value corresponds to that obtained from $\gamma_{\Gamma}$.

To reduce the problem caused by finite data sets, a method of coarse graining has been introduced, with the integrated conditional density $\Gamma^{*}$ (Coleman \& Pietronero 1992):

$\Gamma^{*}=\frac{1}{V} \int_{V} \Gamma \mathrm{d} V$

It produces an artificial smoothing of rapidly varying fluctuations, but correctly reproduces global properties. The integral over $\Gamma$ is strongly related to the correlation integral (Grassberger \& Procaccia 1983),

$C_{2}(r)=\frac{1}{N^{\prime}} \Sigma_{i}\left[\frac{1}{N-1} \Sigma_{j \neq i} \Theta\left(r-\left|\boldsymbol{X}_{i}-\mathbf{X}_{j}\right|\right)\right]$.

$\Theta$ is the Heaviside function. The inner summation is over the whole set of $N-1$ galaxies with coordinates $\boldsymbol{X}_{j}, j \neq i$, and the outer summation is over a subset of $N^{\prime}$ galaxies, taken as centers, with coordinates $\boldsymbol{X}_{i}$. By taking only the inner $N^{\prime}$ galaxies as centers we allow for the effect of the finiteness of the sample, (see e.g. Sylos Labini et al. 1998). This procedure is also widely used in astronomy (Provenzale 1991).

\section{Analytical study of the Einstein-de Sitter case}

As Ribeiro (1992, 1995; hereafter refered to as R92 and R95) and other authors cited therein quite rightly pointed out, galaxy observations are carried out along our past light cone. In a homogeneous FLRW cosmological model, the proper density is constant on hypersurfaces of constant proper time. Therefore, as one looks back to higher and higher redshifted galaxies, the crossing of the null geodesics through hypersurfaces of constant proper time and density implies changes in the observed density and an apparent inhomogeneity can be measured. One can thus wonder whether the inhomogeneous pattern identified by the fractal analysis of galaxy counts is a mere artefact of this light cone effect.

In R95, the magnitude of this effect in an Einstein-de Sitter universe is examined. The conclusion is that "even accepting an error margin of $25 \%$ in the measurements of the global density, a redshift equal to 0.1 is approximately the deepest scale where we could observe a homogeneous distribution of dust in an Einstein-de Sitter model", unimpaired by the light cone effect. Now, such a redshift corresponds to a luminosity distance of about $410 \mathrm{Mpc}$ for a value of the Hubble constant $H_{0}=75 \mathrm{~km} \mathrm{~s}^{-1} \mathrm{Mpc}^{-1}$ and $310 \mathrm{Mpc}$ for $H_{0}=100 \mathrm{~km} \mathrm{~s}^{-1} \mathrm{Mpc}^{-1}$. This is actually less than the depth reached by the ESO Slice Project galaxy survey (Vettolani et al. 1997, 1998), which recently fueled the controversy about the transition towards homogeneity of the luminous matter distribution (Scaramella et al.
1998; Joyce et al. 1999). Other surveys aimed at going beyond this limit are currently under way.

Ribeiro's argument is based on the analysis of the sensitivity of the integrated conditional density $\Gamma^{*}$, Eq. (7), relative to the redshift. We show, in the present section, that the expression retained by Ribeiro for $\Gamma^{*}$ has to be modified, which results in $\Gamma^{*}$ being less sensitive to the redshift value than claimed in R95, thus impairing its conclusions.

In R92, the author establishes expressions for some observational quantities, in an Einstein-de Sitter spacetime, as a function of the radial comoving coordinate $r$, chosen as a parameter along the null geodesics. We list below, and give in R95 units, the quantities relevant for our purpose.

The cumulative number count $N_{\mathrm{c}}(r)$ is the number of sources which lie at radial coordinate distances less than $r$, as seen by the observer at $r=0$. In R95, it is written as

$N_{\mathrm{c}}=\frac{2 r^{3}}{9 M_{\mathrm{G}}}$,

where $M_{\mathrm{G}}$ is the average galactic rest mass.

The luminosity distance $d_{\mathrm{l}}$ of a source is the distance from which the radiating body, if motionless in an Euclidean space, would produce an energy flux equal to the one measured by the observer. It thus verifies

$f=\frac{L}{4 \pi d_{l}^{2}}$,

$L$ being the absolute luminosity, i.e. the luminosity in the rest frame of the source, and $f$ the measured bolometric flux, i.e. integrated over all frequencies by the observer. Its R95 formulation is

$d_{\mathrm{l}}=9 r\left(\frac{2}{3 H_{0}}\right)^{\frac{4}{3}}\left[\left(\frac{18}{H_{0}}\right)^{\frac{1}{3}}-r\right]^{-2}$.

And the redshift $z$ runs as

$1+z=\left(\frac{18}{H_{0}}\right)^{\frac{2}{3}}\left[\left(\frac{18}{H_{0}}\right)^{\frac{1}{3}}-r\right]^{-2}$.

From the definition of $\Gamma$, given in the previous section, one obtains, in Euclidean space-time, (see also Sylos Labini et al. 1998):

$\Gamma(r)=\frac{1}{S} \frac{\mathrm{d} N_{\mathrm{c}}}{\mathrm{d} r}$

where $S$ is the area of a spherical shell of radius $r$.

Arguing that the relevant distance for an analysis performed in a curved space-time is $d_{1}$, Ribeiro defines $\Gamma\left(d_{\mathrm{l}}\right)$ as

$\Gamma\left(d_{\mathrm{l}}\right)=\frac{1}{S} \frac{\mathrm{d} N_{\mathrm{c}}}{\mathrm{d}\left(d_{\mathrm{l}}\right)}$

where $S$ is presented as being the area of the observed spherical shell, of radius $d_{1}$, and is thus written

$S\left(d_{1}\right) \equiv 4 \pi d_{1}^{2}$. 
It is here worth noting that the luminosity distance is the observable quantity relevant for radially measured distances. It is thus correct to use this quantity to evaluate an elementary change in the number density $N_{\mathrm{c}}$ as measured by the observer on his light cone. But, when looking at a cross sectional area $\mathrm{d} S$ perpendicular to the light ray and subtending a solid angle $d \Omega$, the observer must consider the area distance $d_{\mathrm{a}}$, also called "observer area distance" by Ellis (1971) and "corrected luminosity distance" by Kristian \& Sachs (1966), defined by

$\mathrm{d} S=d_{\mathrm{a}}^{2} \mathrm{~d} \Omega$

and related to the luminosity distance of an object measured with the same redshift by

$d_{\mathrm{l}}=d_{\mathrm{a}}(1+z)^{2}$.

Therefore, the area of the observed spherical shell, at a given redshift $z$, used in Eq. (14) must be written

$S=\frac{4 \pi d_{1}^{2}}{(1+z)^{4}}$

From Eqs. (11) and (12), we get an expression for $d_{1}$ as a function of the redshift $z$ :

$d_{\mathrm{l}}(z)=\frac{2}{H_{0}}(1+z-\sqrt{1+z})$.

Using Eqs. (9), (14), (18) and (19), we then derive $\Gamma(z)$ :

$\Gamma(z)=\frac{3 H_{0}^{2}}{8 \pi M_{\mathrm{G}}}\left(\frac{1+z}{2 \sqrt{1+z}-1}\right)$.

As can be easily seen, by comparing Eqs. (15) and (18), this expression differs from the one that would proceed from R95 by a factor $(1+z)^{4}$. Therefore, its sensitivity to the redshift value is much lower.

The volume element $\mathrm{d} V$, relevant for calculations on the light cone, is

$\mathrm{d} V=S \mathrm{~d}\left(d_{1}\right)=4 \pi \frac{d_{1}^{2}}{(1+z)^{4}} \mathrm{~d}\left(d_{1}\right)$.

Substituting Eq. (19) into Eq. (21) and integrating, we obtain

$V=\frac{32 \pi}{H_{0}^{3}} \frac{(\sqrt{1+z}-1)^{3}(2 z+6 \sqrt{1+z}-1)}{15(1+z)^{\frac{5}{2}}}$.

Substituting this expression into Eq. (7) and using Eq. (20), we get

$\Gamma^{*}(z)=\frac{15 H_{0}^{2}}{8 \pi M_{\mathrm{G}}} \frac{1+z}{(2 z+6 \sqrt{1+z}-1)}$

which can be easily compared to Eqs. (19) and (20) in R95. We thus see that, contrary to Ribeiro's claim, $\Gamma^{*}$ differs from the average number density $\langle n\rangle$ if the correct relativistic quantities are retained. It is interesting to note that, as $d_{1}(z), \Gamma^{*}$ is an increasing function of $z$, not a decreasing one, as is Ribeiro's $\langle n\rangle(z)$ function.
Furthermore, if we take temporarily $H_{0}=$ $75 \mathrm{~km} \mathrm{~s}^{-1} \mathrm{Mpc}^{-1}$ to compare our results with those proposed in R95, we see that between $z=0$ and $z=1$ the increase of our $\Gamma^{*}$ is only $5 \%$ (instead of a $87.5 \%$ decrease for Ribeiro's). A significant departure of $\Gamma^{*}$ from its value in an Euclidean universe should therefore occur at redshifts higher than claimed in R95. Its ensuing conclusions thus do not agree with the analysis, which is confirmed by the results of our simulations presented below.

It is also interesting to note that the $5 \%$ increase in our $\Gamma^{*}$ at $z=1$ is markedly less than the $17 \%$ increase of the $d_{\mathrm{l}} / r_{\mathrm{E}}$ ratio, where $r_{\mathrm{E}}=z / H_{0}$ (in units $c=1$ ) is the Euclidean distance. In an Einstein-de Sitter model of the universe, the redshift-distance relation therefore provides a large upper-limit for the departure of $\Gamma^{*}$ from its Euclidean counterpart. The simulations presented below show that this result can be generalized to a wide class of standard homogeneous models of the universe.

\section{The virtual catalog method}

A real catalog gives, for each observed galaxy, a set of four quantities: two angles $\theta$ and $\phi$, the redshift $z$ (or, equivalently, the recession velocity $v$ ) and the apparent luminosity $f$ (or, equivalently, the apparent magnitude $m$ ). This is usually obtained by measuring galaxies with $f>f_{\text {lim }}$ (or, equivalently, $m<m_{\lim }$ ), in a region of the sky delimited by a solid angle $\Omega$.

In an analysis as described in Sect. 2, two of the above observables are transformed, for each galaxy, into nonobservable quantities, the Euclidean distance $r_{\mathrm{E}}$ and the absolute luminosity $L$ (or, equivalently, the absolute magnitude $M$ ), using the following equations:

$r_{\mathrm{E}}=\frac{c z}{H_{0}}$,

$L=4 \pi r_{\mathrm{E}}^{2} f$,

or, equivalently, in Mpc:

$M=m-5 \log _{10} r_{\mathrm{E}}-25$.

Two different kinds of galaxy catalogues are currently available. Pencil beams are generated by deep surveys of a region of the sky delimited by a very narrow solid angle $\Omega$. Much wider distributed samples are generally more limited in depth. A peculiar analysis method applies to each of these two types of datasets.

Owing to their very small orthogonal extension, pencil beam catalogues can only be analysed using the radial count $N_{\mathrm{c}}(<r)$ from the observer (Joyce et al. 1999). As we stressed in Sect. 3, the sensitivity of the cumulative number count $N_{\mathrm{c}}$ to relativistic corrections is well represented by the redshift-luminosity distance relation. In the Einstein-de Sitter model, the departure of this relation from its Euclidean counterpart is only $17 \%$ at $z=1$, which is less than the $25 \%$ error margin in the measurements accepted in R95. The Euclidean approximation can thus be considered as valid up to higher redshifts than 
$z \sim 0.3$, currently reached in these surveys. In the following, we shall not be concerned with the study of relativistc corrections applying to this method, but shall focus our attention on the more accurate one described below.

The second method, in which one averages over a large number of (inner) galaxies, allows us to fit, inside the sample volume, spheres with a sufficiently large maximum radius, since the $\Gamma$ statistic can only be computed up to scales limited by this radius. To avoid selection effects, one generally extracts from the data a set of volume-limited (VL) samples, each sample containing every galaxy with an absolute luminosity below $L_{\mathrm{VL}}$ (or, equivalently, with an absolute magnitude beyond $M_{\mathrm{VL}}$ ) and distance below $R_{\mathrm{VL}}$, such as

$L_{\mathrm{VL}}=4 \pi R_{\mathrm{VL}}^{2} f$

or, equivalently, in Mpc:

$M_{\mathrm{VL}}=m-5 \log _{10} R_{\mathrm{VL}}-25$.

The fractal analysis is thus applied to the set of VL samples (Coleman \& Pietronero 1992; Sylos Labini et al. 1998). Therefore, relativistic corrections must be considered twice. First, for the determination of distances, and second, for the construction of the volume-limited samples extracted from the catalog. As expected, our numerical simulations show that the dominating effect is the distance (or light cone) effect.

We here propose to approach the study of these relativistic corrections from a different point of view, by considering the light cone problem stated as follows.

A homogeneous universe, with a random galaxy distribution on each constant proper time hypersurface, exhibits, when observed on our past light cone, a given pattern of structures (see above Sect. 3). If, for a chosen FLRW model of the universe, we can construct, from a random distribution of points, a virtual catalog as it could be actually observed, we can apply, to this virtual catalog, the above described classical statistical analysis method, in the Euclidean approximation, and consider the fractal dimension obtained. The departure of this dimension from its homogeneous value, $D=3$, provides a measure of the error made when ignoring curvature effects.

In this work, we limit ourselves to the study of spatially flat FLRW models. Spatial flatness seems to be a not too restrictive prescription, as it is compatible with the most recent results of CMBR anisotropy measurements (de Bernardis et al. 2000; Balbi et al. 2000).

The line-element in proper time $t$ and comoving coordinates $r, \theta, \phi$, can thus be written

$\mathrm{d} s^{2}=c^{2} \mathrm{~d} t^{2}-a^{2}(t)\left[\mathrm{d} r^{2}+r^{2}\left(\mathrm{~d} \theta^{2}+\sin ^{2} \theta \mathrm{d} \phi^{2}\right)\right]$

where $a(t)$ is the scale factor which, in the matter dominated area, evolves as $t^{\frac{2}{3}}$.

On each hypersurface $t=$ const., the galaxy distribution is random (homogeneous), with an absolute luminosity distribution given by an assumed luminosity function $\phi(L)$. Galaxies evolve on world lines $(r=$ const., $\theta=$ const., $\phi=$ const.). Thus, if we retain the assumption that, at the distances probed by the current surveys, galaxy evolution can be ignored, a given galaxy, with comoving coordinates $(r, \theta, \phi)$ and absolute luminosity $L$, will keep the same values for these quantities on each $t=$ const. slice.

In the geometrical optics approximation, the light received from the galaxies travels on null geodesics (see e.g Kristian \& Sachs 1966) and thus the objects compiled in the catalogues are all located on our past light cone. At each measured value of the redshift $z$ corresponds an intersection of this light cone with some $t=$ const. hypersurface.

To reconstruct the catalog which would be observed, in a given FLRW universe, by an earth-grounded observer looking on our past light cone from the "here and now" point $\left(t_{0}, r_{0}=0\right)$ to a random distribution of galaxies, we adopt the following method.

First, on the "now" hypersurface $t=t_{0}$, we generate a random distribution of points, i.e. galaxies, fixing, for each of them, values for $r<r_{\max }, \theta<\theta_{\max }, \phi<\phi_{\max }$ and $L>L_{\min }$. Contrary to the producers of real catalogues, we are not limited in angular observation, so we can take the angular maximum values as large as we wish, up to a complete sky. The absolute luminosity distribution is given by the luminosity function retained, see Sect. 5 . This $t=t_{0}$ hypersurface corresponds to a spatial section of a universe which has evolved from different sections $t<t_{0}$ up to $t=t_{0}$, to reach this random configuration.

Then, for each galaxy with a radial comoving coordinate $r$ and absolute luminosity $L$, as first generated on the $t=t_{0}$ hypersurface, we calculate the redshift $z$ and apparent luminosity $f$, as they would be measured on the observer's past light cone, if the chosen FLRW model was to constitute a reliable description of our universe. For this purpose, we use the definition of the luminosity distance:

$f=\frac{L}{4 \pi d_{1}^{2}}$,

where we substitute the expression of $d_{\mathrm{l}}$ as a function of $r$ relevant in the given FLRW model. The redshift $z$ also proceeds from its expression as a function of $r$ in this model (see the Appendix).

We thus obtain the virtual catalog corresponding to a homogeneous galaxy distribution in this model, as a set of quadruplets $(f, z, \theta, \phi)$, each corresponding to a virtually measured galaxy.

Now, we can perform a classical statistical analysis of this virtual catalog, as described in Sect. 2. We can thus obtain, varying the depth of the data sample, different values for the calculated fractal dimension $D$. The departure of $D$ from its homogeneous value, $D=3$, gives the measure of the error made when ignoring curvature effects, at the scale probed by the depth of the retained sample, provided the given cosmological model can be considered as a good approximation of the observed universe. 


\section{Numerical simulations}

\subsection{The method of calculation}

We start with random $(x, y, z)$. Next, we discard all the points for which $R$ is larger than $R_{\max }$. Then, we assign to each point a "Luminosity", $L$, obtained from the distribution:

$$
P(L)=A * \exp (-L) / L \text {. }
$$

We introduce a cut-off at low luminosities (we chose $L=0.05$ ) and at high luminosities (we chose $L=10$.). From $(L, x, y, z)$ we construct, as explained in Sect. 4 and in the Appendix, $(f, z, \phi, \theta)$. For the Einstein-de Sitter case, we use Eqs. (30), (34) and (35). For the parabolic homogeneous models with a non-zero cosmological constant, we use Eqs. (30), (40) and (41). Our choice of the parameters are

$$
\begin{aligned}
& H_{0}=65 \mathrm{~km} \mathrm{~s}^{-1} \mathrm{Mpc}^{-1} \\
& \Omega_{M}=0.3 \\
& \Omega_{\Lambda}=0.7 .
\end{aligned}
$$

Our next stage is to obtain the dimension. We use for that purpose Eq. (8), which is essentially like using $\Gamma^{*}$. As is mentioned in Sect. 2, we have an inner region at a distance larger than some $\mathcal{D}_{\max }$ from the border of the total sample. We now calculate distance and luminosity classically, using the Euclidean formulae:

$$
\begin{aligned}
& r_{\mathrm{E}}=\frac{c z}{H_{0}}, \\
& L_{\mathrm{E}}=L \frac{r_{\mathrm{E}}^{2}}{d_{1}^{2}} .
\end{aligned}
$$

Our limited sample requires $L_{\mathrm{E}}>L_{\mathrm{min}}$.

To understand better our results we perform an additional type of calculation, considering just two points of the inner region, closest to the center and farthest from the center. For each point we can check the local density in addition to the dimension in that region.

\subsection{Results}

For each of the three cosmological models studied, we performed calculations for six different sets of random numbers and took their average. By using these different six catalogues for each cosmological model, we could obtain errors on our results.

We performed the calculations for different $L_{\min }$. For convenience we used a parameter $L_{\mathrm{M}}=$ const $* L_{\min }$.

We then compared our results to those we obtained when ignoring the relativistic corrections for the same random numbers. We compared the dimensions obtained in both cases using a method developed by Benzi et al. (1995). The advantage of this method is that part of systematic errors, like border problems, are less pronounced.

We checked the case $\mathcal{D}_{\max }=200 \mathrm{Mpc}$. Comparing it to the non-relativistic case, no significant difference could be detected.
Table 1. Density results for the three models

\begin{tabular}{ccccc}
\hline Model\# & $P_{\text {RET }}$ & $M 1 / M 2$ & $D\left(R_{\min }\right)$ & $D\left(R_{\max }\right)$ \\
\hline 1 & 100 & $1.35 \pm 0.02$ & $3.01 \pm 0.04$ & $2.97 \pm 0.03$ \\
2 & 100 & $1.12 \pm 0.01$ & $3.00 \pm 0.03$ & $3.00 \pm 0.01$ \\
3 & 100 & $1.00 \pm 0.01$ & $3.00 \pm 0.02$ & $3.00 \pm 0.01$ \\
1 & 25 & $1.41 \pm 0.02$ & $2.99 \pm 0.03$ & $2.99 \pm 0.02$ \\
2 & 25 & $1.29 \pm 0.01$ & $2.98 \pm 0.03$ & $3.01 \pm 0.01$ \\
3 & 25 & $1.22 \pm 0.01$ & $3.00 \pm 0.02$ & $2.97 \pm 0.03$ \\
1 & 8 & $1.46 \pm 0.02$ & $2.99 \pm 0.03$ & $3.01 \pm 0.02$ \\
2 & 8 & $1.42 \pm 0.02$ & $2.97 \pm 0.02$ & $2.99 \pm 0.04$ \\
3 & 8 & $1.40 \pm 0.02$ & $2.99 \pm 0.02$ & $3.01 \pm 0.03$ \\
\hline
\end{tabular}

- Model \#1 is the Einstein - de Sitter Model.

- Model \#2 is the Parabolic Homogenous Model with $\Omega_{\mathrm{M}}=$ $0.3, \Omega_{\Lambda}=0.7$.

- Model \#3 is the Parabolic Homogenous Model with $\Omega_{\mathrm{M}}=$ $0.01, \Omega_{\Lambda}=0.99$.

Checking the differences for the closest and farthest inner point, we obtained that, for the non-relativistic case, 33162 points were inside the sphere, when taking the closest point $\left(R_{\min }=550 \mathrm{Mpc}\right)$. For the farthest point $\left(R_{\max }=1100 \mathrm{Mpc}\right)$, we obtained, in the same sphere, 33152 points. This was expected as we chose a homogeneous sample.

The results are presented in Table 1 . We denote by $M 1$ and $M 2$ the number of points in the two spheres of radius $\mathcal{D}_{\max }$, whose centers are $R_{\min }$ and $R_{\max }$ respectively, and by $P_{\mathrm{RET}}$, the percentage of points retained in each sample, i.e. for three values of $L_{\min }$. We obtain definitely an influence on the average density, depending on the specific model. For the three models, even though this value, $(M 1 / M 2)$, is quite appreciable, still this does not affect the dimension within a $\mathcal{D}_{\max }=200 \mathrm{Mpc}$ radius. For all the cases, we obtain: $D=3.00 \pm 0.03$.

Our next purpose was to check to what extent the dimension diminishes when we go to larger distances. We calculated the case where we consider the whole sphere around our galaxy and take for the inner region a midrange of the radius so that the centers in that region are farther than $\mathcal{D}_{\max }$ from the borders. The calculation was performed just for one case, the Einstein-de Sitter model, and $L_{\text {min }}$ was chosen so that $42 \%$ of the galaxies where retained, on average. We averaged over six sets of 120000 points, chosen randomly, and over all the abovementioned points of the central region (about 12000 ), and checked the decrease in the dimension when averaging over the ranges $150-300 \mathrm{Mpc}$ versus $450-600 \mathrm{Mpc}$. We obtain that the dimension slightly decreases by $1.6 \pm 0.2 \%$, which is consistent with our other calculations.

To compare these weak discrepancies with the light cone effect on the luminosity-distance relation, we note that

- for the Einstein-de Sitter case, at $\mathcal{D}_{\max }=200 \mathrm{Mpc}$, the contribution of the second order term in the power series expansion of $d_{1}$ is a little more than $1 \%$ of the total in Eq. (36), but at $\mathcal{D}_{\max }=600 \mathrm{Mpc}$, it is more than $3 \%$; 
- for the case $\Omega_{\mathrm{M}}=0.3$ and $\Omega_{\Lambda}=0.7$, at $\mathcal{D}_{\max }=$ $200 \mathrm{Mpc}$, this contribution is about $3.2 \%$ of the total in Eq. (42);

- for $\Omega_{\mathrm{M}}=0.01$ and $\Omega_{\Lambda}=0.99$, at $\mathcal{D}_{\max }=200 \mathrm{Mpc}$, it is more than $4 \%$.

We thus see that, except for the Einstein-de Sitter case at $\mathcal{D}_{\text {max }}=200 \mathrm{Mpc}$, this effect is stronger on $d_{1}$ than on $D$. Furthermore, as, at $\mathcal{D}_{\max }=200 \mathrm{Mpc}$, this effect on the fractal dimension $D$ seems to be model independent, the error made by ignoring the relativistic corrections in classical analyses must be smaller, at this scale, than the statistical noise of the method. Therefore, we can safely conclude that, within the studied class of models, the redshift-distance relation can actually be used to provide an upper limit to the relativistic corrections needed to complete this kind of analysis.

Furthermore, as Eq. (17) provides a general relation between $d_{\mathrm{l}}$ and the area distance $d_{\mathrm{a}}$, the smoothing $(1+z)^{4}$ term appears in the calculation of $\Gamma^{*}$ whatever cosmological model is retained to represent the observed universe. We are thus inclined to suggest that the above property could apply to any model and that the averaging procedure has actually some general smoothing result.

\section{Discussion and conclusion}

In the present article, we have studied the effect of curvature on the results of fractal analyses of galaxy distribution.

First, we explored analytically the Einstein-de Sitter model and found that, contrary to the claim in R95, the sensitivity of the integrated conditional density $\Gamma^{*}$ to the redshift is far less than the sensitivity of the luminosity distance. Therefore, in this model, the redshift-distance relation can be used to derive an upper limit to the relativistic corrections applying to classical fractal analyses.

Then, we enlarged our study to a numerical simulation of a wide class of parabolic homogeneous models. To resolve every ambiguity which could arise from a mixing of classical and general relativistic notions of distances, surfaces, volumes and averagings, we calculated the error which could be made by an observer, immersed in a FLRW universe, and analysing, with Euclidean statistical tools, an intrinsic homogeneous pointlike galaxy distribution, as measured on his past light cone. We have shown that, in these models and up to distances far beyond those probed by the current analyses, the curvature effect is negligible compared to the measurement uncertainties. For $H_{0}=$ $65 \mathrm{~km} \mathrm{~s}^{-1} \mathrm{Mpc}^{-1}$, the three tested models exhibit, at the scale of $200 \mathrm{Mpc}$, a discrepancy for the numerically calculated fractal dimension of on the order of $1 \%$, and a $2 \%$ limit still holds for the Einstein-de Sitter case at scales on the order of 450-600 Mpc.

In fact, contrary to the author's claim in R95, the procedure averaging over many points, far from increasing the curvature effect, has some smoothing results, and therefore this effect is weaker on the fractal dimension than on e.g. luminosity distances. We have actually proved this property for the class of universes under study and have given grounds to suppose that it can apply to any other cosmological model.

Therefore, as regards the current and forthcomimg results which could be derived from Euclidean statistical analyses of galaxy data, the relativistic corrections could be consistently ignored, up to the abovementioned scales, and probably far beyond, in every universe for which the dependence of the luminosity distance on the redshift does not too widely depart from the Hubble law at the probed scales.

We want to mention, for completeness, that the curvature correction is not the only bias that can impair the results of such analyses. Evolution effects and, mainly, Kcorrections are known to generate other kinds of errors, but are beyond the scope of the present work. For a discussion of the impact of K-corrections on such an issue, we refer the interested reader to Scaramella et al. (1998) and Joyce et al. (1999).

Acknowledgements. Part of this work was performed while one of us (R.T.) was on a sabbatical leave at the Observatoire de Paris-Meudon. He wishes to thank Brandon Carter and Nathalie Deruelle for their hospitality. The authors want to thank also the referee for accurate remarks which led them to add interesting developments to this work.

\section{Appendix A: The FLRW models tested}

We give, in this appendix, the expressions for $d_{\mathrm{l}}$ and $z$ retained for the conversion of the $(L, r, \theta, \phi)$ data into the $(f, z, \theta, \phi)$ quadruplets, in the FLRW models chosen to perform the numerical simulations described in Sect. 5. We have limited these simulations to three examples of the spatially flat subclass: the Einstein-de Sitter case, with $\Omega_{\mathrm{M}}=1$ and $\Omega_{\Lambda}=0$, currently the most popular model with $\Omega_{\mathrm{M}}=0.3$ and $\Omega_{\Lambda}=0.7$ and a low matter density case, with $\Omega_{\mathrm{M}}=0.01$ and $\Omega_{\Lambda}=0.99$. As it can be easily seen from a comparison of the $d_{1}$ expansions in powers of $z$ calculated below, the low matter density case exhibits the larger second order term correction for "realistic" flat models with $0<\Omega_{\mathrm{M}} \leq 1$. It can thus been considered as a limiting case for the study presented here.

For the Einstein-de Sitter case, we use Ribeiro's relations in R95, corrected for consistency from a dimensional point of view:

$$
\begin{aligned}
& d_{1}=r\left(1-\frac{H_{0}}{2 c} r\right)^{-2} \\
& 1+z=\left(1-\frac{H_{0}}{2 c} r\right)^{-2}
\end{aligned}
$$

The power series expansion of $d_{1}$ thus reads

$$
d_{1}=\frac{c z}{H_{0}}+\frac{c z^{2}}{4 H_{0}}+\mathcal{O}\left(z^{3}\right)
$$


For the parabolic homogeneous model with a non-zero cosmological constant, the following expressions can be found in text books (see e.g. Carroll et al. 1992):

$d_{\mathrm{l}}=(1+z) r$,

$$
\begin{aligned}
d_{1}= & \frac{c(1+z)}{H_{0}} \\
& \times \int_{0}^{z}\left[\left(1+z^{\prime}\right)^{2}\left(1+\Omega_{\mathrm{M}} z^{\prime}\right)-z^{\prime}\left(2+z^{\prime}\right) \Omega_{\Lambda}\right]^{-\frac{1}{2}} \mathrm{~d} z^{\prime}
\end{aligned}
$$

It is easy to derive an exact expression for $r$ from Eqs. (37) and (38):

$r=\frac{c}{H_{0}} \int_{0}^{z}\left[\left(1+z^{\prime}\right)^{2}\left(1+\Omega_{\mathrm{M}} z^{\prime}\right)-z^{\prime}\left(2+z^{\prime}\right) \Omega_{\Lambda}\right]^{-\frac{1}{2}} \mathrm{~d} z^{\prime}$,

For small $z$, we can Taylor expand the right-hand side of Eq. (39) and invert to obtain

$z=\frac{2}{2 \Omega_{\Lambda}-\Omega_{\mathrm{M}}-2}\left(\sqrt{1+\frac{H_{0}}{c}\left(2 \Omega_{\Lambda}-\Omega_{\mathrm{M}}-2\right) r}-1\right)$.

Substituting Eq. (40) into Eq. (37), we get $d_{\mathrm{l}}$ as a function of $r$ :

$$
\begin{aligned}
d_{\mathrm{l}}= & r+\frac{2 r}{2 \Omega_{\Lambda}-\Omega_{\mathrm{M}}-2} \\
& \times\left(\sqrt{1+\frac{H_{0}}{c}\left(2 \Omega_{\Lambda}-\Omega_{M}-2\right) r}-1\right),
\end{aligned}
$$

and can derive the power series expansion of $d_{1}$ :

$d_{\mathrm{l}}=\frac{c z}{H_{0}}+\frac{c}{4 H_{0}}\left(2-\Omega_{\mathrm{M}}+2 \Omega_{\Lambda}\right) z^{2}+\mathcal{O}\left(z^{3}\right)$.

\section{References}

Bahcall, N. A., Cen, R., Davé, R., et al. 2000, to be published in ApJ, 541, [astro-ph/0002310]

Balbi, A., Ade, P., Bock, J., et al. to be published in ApJL, [astro-ph/0005124]

Benzi, R., Ciliberto, S., Baudet, C., \& Chavarria, G. R. 1995, Physica D, 80, 385

Carroll, S. M., Press, W. H., \& Turner, E. L. 1992, ARA\&A, 30, 499

Célérier, M. N. 2000a, A\&A, 362, 840

Célérier, M. N. 2000b, A\&A, 353, 63

Charlier, C. V. L. 1908, Arkiv. Mat. Astron. Fys., 4, 1
Charlier, C. V. L. 1922, Arkiv. Mat. Astron. Fys., 16, 1

Clarkson, C. A., \& Barrett, R. K. 1999, Class. Quantum Grav., 16,3781

Coleman, P. H., \& Pietronero, L. 1992, Phys. Rep., 231, 311

Davis, M., \& Peebles, P. J. E. 1983, ApJ, 267, 465

de Bernardis, P., Ade, P. A. R., Bock, J. J., et al. 2000, Nature, 404,955

de Laix, A. A., \& Starkman, G. D. 1998, [astro-ph/9802270]

de Vaucouleurs, G. 1953, AJ, 58, 30

de Vaucouleurs, G. 1970, Sci., 167, 1203

Ehlers, J., Geren, P., \& Sachs, R. K. 1968, J. Math. Phys., 9, 1344

Ellis, G. F. R. 1971, in General Relativity and Cosmology., ed. R. K. Sachs, Rend. Scuola Int. Fis. Enrico Fermi, XLVII Corso (Academic Press, New York)

Grassberger, P., \& Procaccia, I. 1983, Phys. Rev. Lett., 50, 346

Humphreys, N. P., Matravers, D. R., \& Maartens, R. 1998, Class. Quantum Grav., 15, 3041

Joyce, M., Montuori, M., Sylos Labini, F., \& Pietronero, L. 1999, A\&A, 344, 387

Kerscher, M. 1999, A\&A, 343, 333

Kristian, J., \& Sachs, R. K. 1966, ApJ, 143, 379

Kurki-Suonio, H., \& Liang, E. 1992, ApJ, 390, 5

Mandelbrot, B. B. 1982, The Fractal Geometry of Nature (Freeman, San Francisco)

Mansouri, R., \& Rahvar, S. 1999, [astro-ph/9907041]

Martinez, V. J. 1999, Sci., 284, 445

Murante, G., Provenzale, A., Spiegel, E. A., \& Thieberger, R. 1998, Ann. New York Acad. Sci., 867, 258

Mustapha, N., Bassett, B. A. C. C., Hellaby, C., \& Ellis, G. F. R. 1998, Class. Quantum Grav., 15, 2363

Peebles, P. J. E. 1980, Large Scale Structure of the Universe (Princeton University Press, Princeton)

Pietronero, L. 1987, Physica A, 144, 257

Provenzale, A. 1991, in Applying Fractals in Astronomy, ed. A. Heck, \& J. M. Perdang (Springer, Berlin)

Ribeiro, M. B. 1992, ApJ, 395, 29

Ribeiro, M. B. 1995, ApJ, 441, 477

Scaramella, R., Guzzo, L., Zamorani, G., et al. 1998, A\&A, 334,404

Stoeger, W. R., Maartens, R., \& Ellis, G. F. R. 1995, ApJ, 443, 1

Sylos Labini, F., Montuori, M., \& Pietronero, L. 1998, Phys. Rep., 293, 61

Thieberger, R., Spiegel, E. A., \& Smith, L. A. 1990, in The Ubiquity of Chaos, ed. S. Krasner, AAAS Press

Vettolani, G., Zucca, E., Zamorani, G., et al. 1997, A\&A, 325, 954

Vettolani, G., Zucca, E., Merighi, R., et al. 1998, A\&AS, 130, 323 